\title{
Fast Timing Analysis of Plane Circuits via Two-Layer CNN-based Modeling
}

\author{
Yuichi Tanji \\ Kagawa University \\ tanji@eng.kagawa-u.ac.jp
}

\author{
Hideki Asai \\ Shizuoka University \\ hideasai@sys.eng.shizuoka.ac.jp
}

\author{
Masayoshi Oda, Yoshifumi Nishio \\ Tokushima University \\ nishio@ee.tokushima-u.ac.jp
}

\author{
Akio Ushida \\ Tokushima Bunri University \\ ushida@fe.bunri-u.ac.jp
}

\begin{abstract}
A fast timing analysis of plane circuits via two-layer CNNbased modeling, which is necessary for the solution of power/signal integrity problems in printed circuit boards and packages, is presented. Using the new notation expressed by the two-layer CNN, more than 1500 times faster simulation is achieved, compared with Berkeley SPICE (ngspice). In CNN community, CNNs are generally simulated by explicit numerical integration algorithms such as the forward Euler and RungeKutta methods. However, since the system of the two-layer CNN becomes stiff, we cannot analyze the $\mathrm{CNN}$ by using an explicit numerical integration algorithm. Hence, to analyze the two-layer CNN and reduce the computational cost, the leapfrog method is introduced in this paper. This procedure would open a CNN application up to electronic design automation area.
\end{abstract}

\section{INTRODUCTION}

VLSI implementations, potential applications for image processing, and nonlinear wave phenomena of CNNs have attracted many interests in the last two decades. As one of the studies, the nonlinear wave phenomena have been analyzed via CNNs [1], [2], [3], where a discrete model of partial differential equation is expressed by $\mathrm{CNN}$ and the CNN universal machine [4] simulates the phenomena quickly using the features of custom hardware. In viewing the CNN simulations from engineering application sides, the ability of solving partial differential equations quickly is very attractive and there may be various applications.

In this paper, we analyze the plane circuits using CNN, which is necessary for ensuring power/signal integrity of VLSIs, packages, and printed circuit boards in the high-speed electronic systems [5], [6]. To address the issue of signal/power integrity, electromagnetic field should be analyzed. Then, the discrete models of the Maxwell's or Helmholtz's equations involve the inductance effects, whereas almost of CNNs do not have the inductance effects. Hence, we introduce the two-layer CNN [7] to model the plane circuits using the coupling templates.

The CNN universal machine simulates CNNs by the forward Euler method. However, the linear passive circuits as a model of VLSI, package, printed circuit board are stiff, which prohibits the use of the explicit numerical integration methods such as forward Euler method. Alternatively, the implicit numerical integration methods such as backward Euler, backward difference, and Gear methods [8], which are used in SPICE-like simulators, are applicable to solving the stiff problems, but these methods are computationally inefficient since they need to solve a set of equations simultaneously at each time point. To reduce the computational costs, we introduce the leapfrog method [9], [10], where the state vectors in the first and second layers are decoupled and updated by turns in the manner as the forward Euler method. This method is a derivative of FDTD method [11] which is known as a Maxwell's solver. In the illustrative examples, it is shown that the proposed procedure via the two-layer CNN-based modeling is 1,553 times faster than Berkely SPICE (ngspice). This means that we can expect the further speed up if these computations are carried out on a hardware accelerator as the CNN universal machine.

\section{Two-Layer CNN-BAsed Modeling of Plane Circuits}

Ensuring the signal/power integrity of packages and printed circuit boards in high-speed electronic systems needs the analysis of plane circuits, where the physical phenomena are governed by the Helmholtz's equations which are the Maxwell's equations on Lorentz or Coulomb gauge. However, since simulating the Helmholtz's equations requires huge CPU costs, the plane circuits are approximated by linear passive R, L, C, and G elements shown in Fig. 1 [12]. The normalized KCL equation at the node and KVL one of the branch consisting of a resistor $\mathrm{R}$ and an inductor $\mathrm{L}$ are represented as follows,

$$
\begin{array}{r}
\frac{d v_{i, j}(t)}{d t}=-G \sqrt{\frac{L}{C}} v_{i, j}(t)+\sqrt{\frac{L}{C}} i_{i, j}(t) \\
\frac{d i_{i, j}(t)}{d t}=-R \sqrt{\frac{C}{L}} i_{i, j}(t)+\sqrt{\frac{C}{L}}\left(v_{i-1, j}(t)\right. \\
\left.+v_{i+1, j}(t)+v_{i, j-1}(t)+v_{i, j+1}(t)-4 v_{i, j}(t)\right)
\end{array}
$$

where

$$
t=\frac{1}{\sqrt{L C}} \tau
$$

and $\tau$ and $t$ show the actual and normalized times, respectively.

Let us consider expressing (1) and (2) by the two-layer CNN shown in Fig. 2. The state equations of the two-layer CNN are written by

$$
\begin{aligned}
\frac{d v_{x 1 i j}(t)}{d t} & =-v_{x 1 i j}(t)+\sum_{\mathcal{C}(k, l) \in N_{r}(i, j)} A_{1}(i, j ; k, l) v_{y 1 k l}(t) \\
& +\sum_{\mathcal{C}(k, l) \in N_{r}(i, j)} B_{1}(i, j ; k, l) v_{u 1 k l}(t) \\
& +\sum_{\mathcal{C}(k, l) \in N_{r}(i, j)} C_{1}(i, j ; k, l) v_{y 2 k l}(t)+I_{1}, \\
\frac{d v_{x 2 i j}(t)}{d t} & =-v_{x 2 i j}(t)+\sum_{\mathcal{C}(k, l) \in N_{r}(i, j)} A_{2}(i, j ; k, l) v_{y 2 k l}(t) \\
& +\sum_{\mathcal{C}(k, l) \in N_{r}(i, j)} B_{2}(i, j ; k, l) v_{u 2 k l}(t) \\
& +\sum_{\mathcal{C}(k, l) \in N_{r}(i, j)} C_{2}(i, j ; k, l) v_{y 1 k l}(t)+I_{2},
\end{aligned}
$$

where $N_{r}(i, j)$ is $r$-neighborhood of cell $\mathcal{C}(i, j)$ as $N_{r}(i, j)=$ $\left\{\mathcal{C}(k, l) \mid \max \{|k-i|,|l-j|\} \leq r . v_{x 1 i j}(t), v_{y 1 i j}(t)\right.$, and $v_{u 1 i j}(t)$ are the internal state, the output, the input of a cell $\mathcal{C}(i, j)$ in the first layer $\mathrm{CNN}$, respectively. $v_{x 2 i j}(t), v_{y 2 i j}(t)$, and $v_{u 2 i j}(t)$ are the internal state, the output, the input of a cell $\mathcal{C}(i, j)$ in the second layer. $A_{m}(i, j ; k, l), B_{m}(i, j ; k, l)$, and $C_{m}(i, j ; k, l)$ are the feedback, 


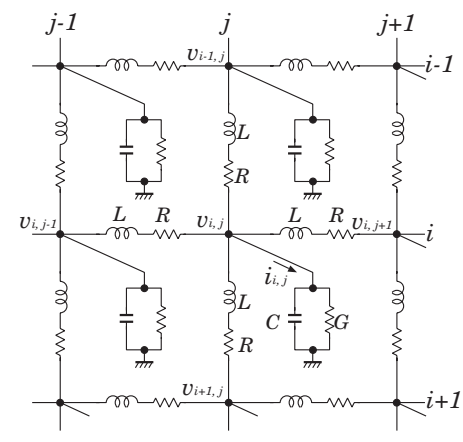

Fig. 1. Circuit model of plane circuit.

control, coupling templates in the $m$-th layer $\mathrm{CNN}$, respectively [7]. The output functions of the two-layer $\mathrm{CNN}$ are described by

$$
\begin{aligned}
& v_{y 1 i j}(t)=\frac{1}{2}\left(\left|v_{x 1 i j}(t)+1\right|-\left|v_{x 1 i j}(t)-1\right|\right), \\
& v_{y 2 i j}(t)=\frac{1}{2}\left(\left|v_{x 2 i j}(t)+1\right|-\left|v_{x 2 i j}(t)-1\right|\right) .
\end{aligned}
$$

Comparing the circuit equations (1) and (2) with the CNN ones (4) and (5), we can describe the cloning templates for the plane circuits as

$$
\begin{aligned}
& \boldsymbol{A}_{1}=\left(\begin{array}{ccc}
0 & 0 & 0 \\
0 & 1-G \sqrt{\frac{L}{C}} & 0 \\
0 & 0 & 0
\end{array}\right), \boldsymbol{B}_{1}=\mathbf{0}, \\
& \boldsymbol{C}_{1}=\left(\begin{array}{ccc}
0 & 0 & 0 \\
0 & \sqrt{\frac{L}{C}} & 0 \\
0 & 0 & 0
\end{array}\right), I_{1}=0, \\
& \boldsymbol{A}_{2}=\left(\begin{array}{ccc}
0 & 0 & 0 \\
0 & 1-R \sqrt{\frac{L}{C}} & 0 \\
0 & 0 & 0
\end{array}\right), \boldsymbol{B}_{2}=\mathbf{0}, \\
& \boldsymbol{C}_{2}=\left(\begin{array}{ccc}
0 & \sqrt{\frac{L}{C}} & 0 \\
\sqrt{\frac{L}{C}} & -4 \sqrt{\frac{L}{C}} & \sqrt{\frac{L}{C}} \\
0 & \sqrt{\frac{L}{C}} & 0
\end{array}\right), I_{2}=0,
\end{aligned}
$$

where the matrices $\boldsymbol{A}_{m}, \boldsymbol{B}_{m}$, and $\boldsymbol{C}_{m}$ are the matrix representation of $A_{m}(i, j ; k, l), B_{m}(i, j ; k, l)$, and $C_{m}(i, j ; k, l)$, respectively. Since the plane circuit shown in Fig. 1 is linear, the output functions (6) and (7) are redefined by the linear ones as

$$
\begin{aligned}
& v_{y 1 i j}(t)=v_{x 1 i j}(t), \\
& v_{y 2 i j}(t)=v_{x 2 i j}(t) .
\end{aligned}
$$

\section{LEAPFROG METHOD}

In the CNN universal machine [4], the dynamics of CNN is simulated by using the forward Euler method. However, if the circuit is stiff, the forward Euler method may diverge during the transient analysis. For example, consider an RLC series circuit. The admittance $Y(s)$ of the circuit is given by

$$
Y(s)=\frac{(1 / L) s}{s^{2}+(R / L) s+1 / L C} .
$$

The poles of (12) are approximated by $j(1 / \sqrt{L C})$ and $-(R / L)$. Using the typical parameters $R=1.73 \times 10^{-3}[\Omega], L=2.26 \times$ $10^{-9}[H]$, and $C=9.15 \times 10^{-15}[F]$ for the package [6], we obtain

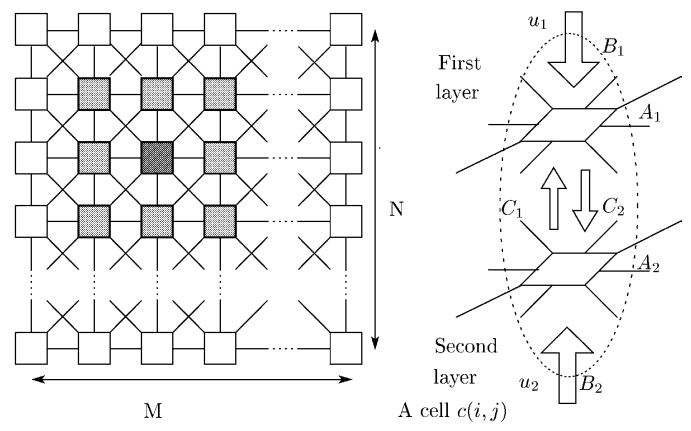

Fig. 2. Two-Layer CNN.

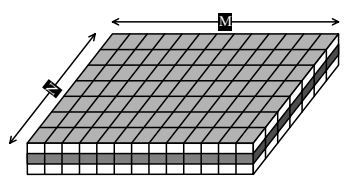

(a)

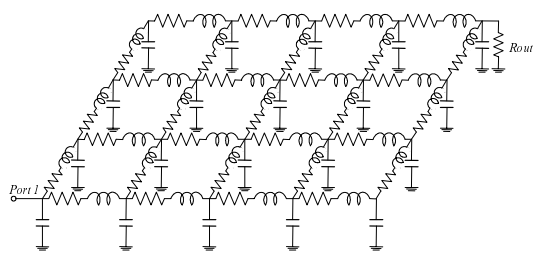

(b)

Fig. 3. Modeling of plane circuit. (a)Example plane circuit. (b)Equivalent circuit.

the approximated poles $2.10 \times 10^{11}$ and $7.61 \times 10^{5}$, which implies the RLC series circuit is stiff and the forward Euler method used in the $\mathrm{CNN}$ universal machine is not sufficient for solving this problem. We simulated the two-Layer CNN corresponding to the plane circuit shown in Fig. 3(a), where the circuit is modeled by $3 \times 3$ cells and approximated by the RLC circuit shown in Fig. 3(b). Figure 4(a) shows the transient voltage waveform on the output resister $R_{\text {out }}$ in Fig. 3(b) when a pulse waveform is given at "Port1", where the transient waveform was calculated by the forward Euler method. For a comparison, the result is compared with one obtained from Berkeley SPICE. The response obtained from the forward Euler method is vibrated violently and the simulation will break down obviously.

Although the nonlinear wave propagation phenomena of CNNs are one of the attractive fields of CNN [2], the conventional forward Euler method or the Runge Kutta method is not necessarily applicable to all the cases as shown in this section. Instead of the explicit method, we can use an implicit numerical integration method such as backward Euler, backward difference, and Gear methods [8]. However, these methods need to solve a set of linear equations simultaneously, which prohibits the application of these methods to the large scale problems. To analyze the large scale and stiff circuits, we use the leapfrog method [10] which is a derivative of FDTD method [11] for circuit simulation [9].

From (10) and (11), a state in the first and second layer is equal to the output itself. As shown in Fig. 2, the first and second layers interact each other through the coupling templates $C_{1}(i, j ; k, l)$ of (4) and $C_{2}(i, j ; k, l)$ of (5). Therefore, the first and second layers 


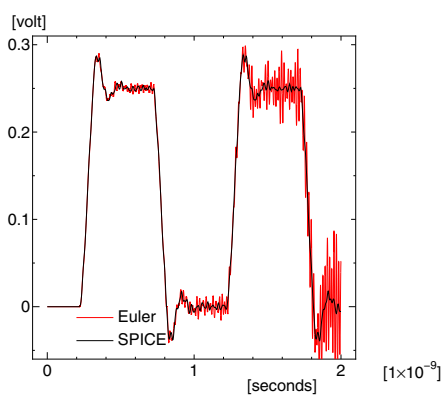

(a)

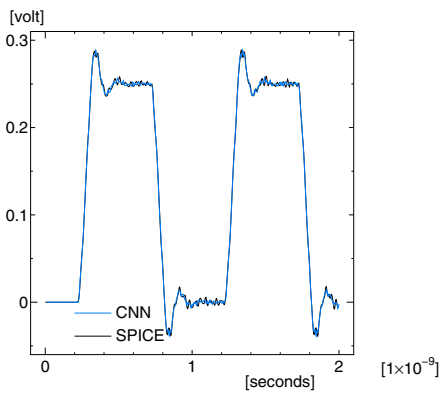

(b)

Fig. 4. Timing analysis results obtained by (a)forward Euler method (b)leapfrog method.

of the $\mathrm{CNN}$ are coupled through the state vectors in both layers. These relations appear in $v_{x 1 i j}(t)$ and $v_{y 2 k l}(t)$ of (4) or $v_{x 2 i j}(t)$ and $v_{y 1 k l}(t)$ of (5).

In the leapfrog method, two coupled state vectors are alternately solved. Hence, the updated timing of the first layer is being shifted with half time step to the second layer. On the other hand, when the forward Euler method is used for simulating the $\mathrm{CNN}$, the state vectors in both layers are updated simultaneously as shown in Fig. 5 .

The update rules of the leapfrog method for the two-layer CNN are described by

$$
\begin{gathered}
\frac{v_{x 1 i j}\left(t_{i+1}\right)-v_{x 1 i j}\left(t_{i}\right)}{t_{i+1}-t_{i}}=-v_{x 1 i j}\left(t_{i+1}\right) \\
+\sum_{\mathcal{C}(k, l) \in N_{r}(i, j)} A_{1}(i, j ; k, l) v_{y 1 k l}\left(t_{i+1}\right) \\
+\sum_{\mathcal{C}(k, l) \in N_{r}(i, j)} B_{1}(i, j ; k, l) v_{u 1 k l}\left(t_{i+1 / 2}\right) \\
+\sum_{\mathcal{C}(k, l) \in N_{r}(i, j)} C_{1}(i, j ; k, l) v_{y 2 k l}\left(t_{i+1 / 2}\right)+I_{1}, \\
\frac{v_{x 2 i j}\left(t_{i+1 / 2}\right)-v_{x 2 i j}\left(t_{i-1 / 2}\right)}{t_{i+1 / 2}-t_{i-1 / 2}}=-v_{x 2 i j}\left(t_{i+1 / 2}\right) \\
+\sum_{\mathcal{C}(k, l) \in N_{r}(i, j)} A_{2}(i, j ; k, l) v_{y 2 k l}\left(t_{i+1 / 2}\right) \\
+\sum_{\mathcal{C}(k, l) \in N_{r}(i, j)} B_{2}(i, j ; k, l) v_{u 2 k l}\left(t_{i}\right) \\
+\sum_{\mathcal{C}(k, l) \in N_{r}(i, j)} C_{2}(i, j ; k, l) v_{y 1 k l}\left(t_{i}\right)+I_{2} .
\end{gathered}
$$

Since the state vectors are alternately updated, we do not have to solve
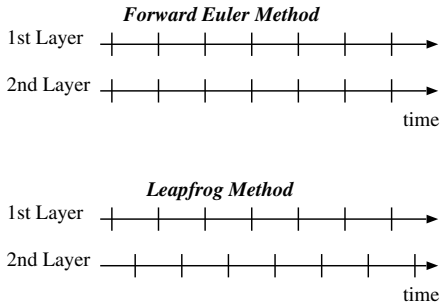

Fig. 5. Updated Timing of the state vectors in the forward Euler and leapfrog methods.

a set of linear equations at each time point, differently from the case of applying an implicit numerical integration method. As a result, the leapfrog method is much faster than the SPICE simulation which uses an implicit numerical integration method for timing analysis.

We simulated the example of $3 \times 3$ cells using the leapfrog method. Figure 4(b) shows the transient voltage waveform on the output resister $R_{\text {out }}$ shown in Fig. 3(b). To obtain the result, the time step size is taken as 100 times larger than the forward Euler method in the case of Fig. 4(a), which means that the leapfrog method is robust for a stiff problem, though any implicit method is not used.

It should be noted that the leapfrog method is restricted by the Courant condition which gives us the allowable largest time step size $h$ in order to ensure the stability. The Courant condition is obtained as [9]

$$
h \leq \sqrt{L_{\min } C_{\min }},
$$

where $L_{\min }$ and $C_{\min }$ are minimum inductance and capacitance, respectively. If the normalized time of (3) is $\tau=1 / \sqrt{L_{\min } C_{\min }}$, the Courant condition becomes $t_{i+1}-t_{i} \leq 1$. Therefore, putting the time step size as $t_{i+1}-t_{i}=1$, we can obtain the discrete time model, which is equivalent to the Discrete Time CNN (DT-CNN) [13], [14], of the two-layer CNN by (13) and (14).

\section{EXAMPLES}

To show the performance of the proposed method via two-layer CNN-based modeling, the plane circuit shown in Fig. 3(a) was simulated. The plane circuit is divided into identical cells (hexahedrons) which are modeled by an RLC $\pi$-model [6], and the equivalent circuit is obtained as Fig. 3(b). Based on the electromagnetic theory, the parameters of the RLC $\pi$-model corresponding to the VLSI package are determined as $R=1.73 \times 10^{-3}[\Omega], L=2.26 \times 10^{-9}[H]$, and $C=9.15 \times 10^{-15}[F][6]$.

We calculated the transient responses of the $\mathrm{CNN}$ by the leapfrog method and compared with Berkeley SPICE (ngspice) from the computational cost point of view. The $\mathrm{CNN}$ simulations were carried out by Matlab 7 on Federa Core 3. The CPU times of CNN and SPICE simulations were measured on Pentium 4 with $3 \mathrm{GHz}$ clock and 1 GByte memory.

The linear circuit shown in Fig. 3(b) was driven by a current source of 0.1 [ns] fall/rise time, 0.4 [ns] pulse width, 0.2 [ns] delay time, 1.0 [ns] period, and $5[\mathrm{~mA}]$ amplitude at "Port1". The transient analysis was carried out for the time interval from 0 [ns] to 2.0 [ns] using 1 [ps] time step size. Table I shows the comparison of CPU times. For $320 \times 320$ cells, the proposed method via the twolayer $\mathrm{CNN}$-based modeling is 1,553 times faster than ngspice. To investigate the accuracy of the simulation results, we calculated the transient waveforms for the case of $10 \times 10$ cells using ngspice with a variety of time step sizes. Figures 6(a) and 6(b) are the transient 
TABLE I

COMPARISON OF CPU TIMES FOR A PLANE CIRCUIT.

\begin{tabular}{|c|c||c|c|c|}
\hline cells & elements & CNN & ngspice & Speed-up \\
\hline $10 \times 10$ & 341 & 0.53 & 2.33 & 4.4 \\
\hline $20 \times 20$ & 1281 & 0.74 & 19.567 & 26.44 \\
\hline $40 \times 40$ & 4961 & 1.79 & 155.56 & 86.90 \\
\hline $80 \times 80$ & 19521 & 6.25 & 1400.10 & 224.02 \\
\hline $160 \times 160$ & 77441 & 32.68 & 16535.75 & 505.99 \\
\hline $320 \times 320$ & 308481 & 152.09 & 236199.93 & 1553.03 \\
\hline
\end{tabular}

[V]

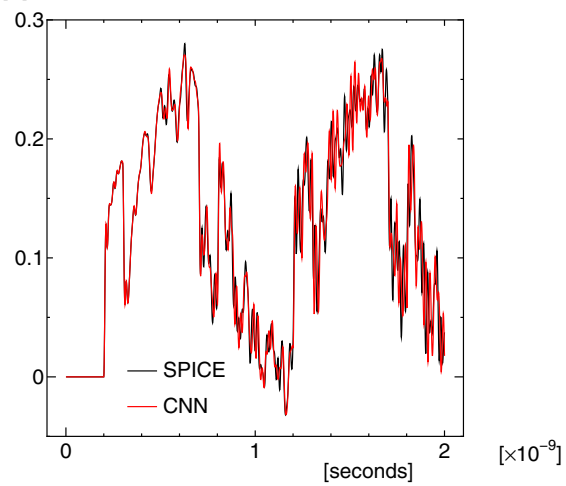

(a)

[V]

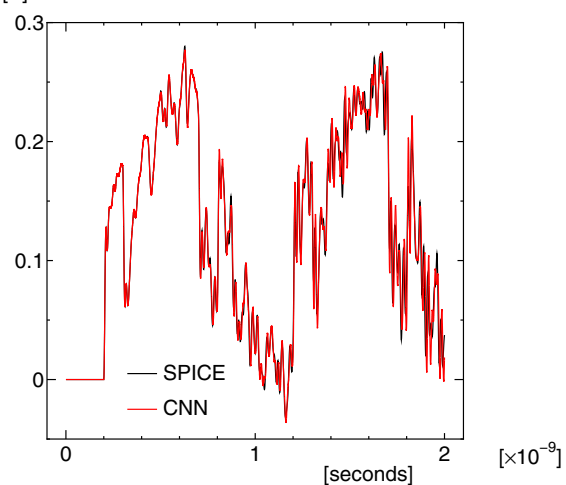

(b)

Fig. 6. Transient responses calculated by SPICE with (a) $h=1[\mathrm{ps}]$ and (b) $h=0.01$ [ps], where the time step size in the CNN simulation is fixed with $h=1$ [ps].

voltage waveforms at "Port1" using ngspice with 1 [ps] and 0.01[ps] time step sizes, respectively. Although "Port1" is driven by a current source having a pulse waveform, the voltage waveform at "Port1" is largely degraded. It should be noted that the time step size of the proposed method via two-layer CNN is fixed by 1 [ps]. We can see that the simulation results by the proposed method is more accurate than the ones by SPICE. Therefore, if the CPU times are evaluated with the same accuracy, the computational efficiency of the proposed method becomes more remarkable than that listed in Tab. I. In this example, we do not consider any parallelism of CNN. Thus, if a hardware accelerator for simulating the two-layer $\mathrm{CNN}$ is realized, further speed-up is expected.

\section{CONCLUSiOnS}

In this paper, a fast timing analysis of the plane circuits using the CNN-based modeling has been presented, where the Maxwell's equations of plane circuits are expressed by the two-layer $\mathrm{CNN}$ and the dynamics of the two-layer $\mathrm{CNN}$ are alternately calculated by the leapfrog method. We can assume that these computations are carried out on a hardware accelerator as the CNN universal machine. Since the proposed method is sufficiently fast on a single processor, the CNN simulations on the hardware accelerator might be extremely fast. The leapfrog method is based on the FDTD method [11]. Therefore, the hardware accelerator would be extended to full wave analysis of electromagnetic field.

\section{REFERENCES}

[1] T. Roska, L. O. Chua, D. Wolf, T. Kozek, R. Tetzlaff, and F. Puffer, "Simulating nonlinear waves and partial differential equations via cnnpart i," IEEE Trans. on Circuits Syst., vol. 42, no. 10, pp. 807-815, 10 1995.

[2] L. O. Chua, "Passivity and complexity," IEEE Trans. Circuits Syst.-I, vol. 46, pp. 71-82, 1999.

[3] T. Roska, L. O. Chua, D. Wolf, T. Kozek, R. Tetzlaff, and F. Puffer, "Simulating nonlinear waves and partial differential equations via cnnpart ii," IEEE Trans. on Circuits Syst., vol. 42, no. 10, pp. 816-820, 1995.

[4] L. O. Chua and T. Roska, "The cnn universal machine," IEEE Trans. Circuits Syst.-II, vol. 40, pp. 163-173, 1993.

[5] M. W. Beattie and L. T. Pileggi, "Modeling magnetic coupling for onchip interconnect," Proc. Design Automation Conference 2001, 2001.

[6] T. Watanabe, K. Srinivasan, H. Asai, and M. Swaminathan, "Modeling of power distribution networks with retardation using the transmission matrix method," Proc. EPEP'04, 2004.

[7] Z. Yang, Y. Nishio, and A. Ushida, "Generation of various types of spatio-temporal phenomena in two-layer cellular neural networks," IEICE Trans. Fundamentals, vol. E87-A, no. 4, pp. 864-871, 2004.

[8] L. O. Chua and P.-M. Lin, Computer-Aided Analysis of Electrical Circuits: Algorithms and Computational Techniques. Englewood Cliffs: Prentice-Hall, Inc., 1975.

[9] J. E. Schutt-Aine, "Latency insertion method (lim) for the fast transient simulation of large networks," IEEE Trans. Circuits Syst.-I, vol. 48, pp. 81-89, 2001.

[10] H. Kubota, Y. Tanji, T. Watanabe, and H. Asai, "Generalized method of the time-domain circuit simulation based on lim with mna formulation," Proc. Custom Integrated Circuits Conference, 2005.

[11] K. S. Yee, "Numerical solution of intial boundary value problems involving maxwell's equations in isotrophic media," IEEE Trans. Antennas Propagat., vol. 14, no. 4, pp. 302-307, 1966.

[12] N. Na, J. Choi, S. Chun, and M. Swaminathan, "Modeling and transient simulation of planes in electromagnetic packages," IEEE Trans. Comp., Packag., Manufact. technol. B, vol. 21, pp. 157-163, 1998.

[13] H. Harrer and J. T. Nossek, "Discrete-time cellular neural networks," Int. J. Circuit Theory and Applications, vol. 20, pp. 453-467, 1992.

[14] M. Ikegami and M. Tanaka, "Image coding and decoding by discrete time cellular neural networks," IEICE Trans., vol. J77-A, pp. 954-964, 1994.

[15] [Online]. Available: http://ngspice.sourceforge.net/ 